\title{
Controllingerfolg - Macht Controlling erfolgreich, und wenn ja wie?
}

Erfolg ist ein ureigenes Controllerthema: Die Frage im Kopf zu haben, ob sich etwas rechnet, lässt sich geradezu als ein genetischer Code von Controllern bezeichnen. Allerdings stellen sie die Frage für sich selbst nur selten und zumeist nicht wirklich konsequent. Welcher Controller kann seinem Manager schon "aus dem Stand" seinen Erfolgsbeitrag belegen?

Das Thema ist für das Controlling essentiell. Schon aus Hygienegründen sollten Controller nicht von anderen fordern, was sie selbst nicht leisten. Controller könnten weiterhin mit dem Beleg ihres Erfolgs dem häufig zu vernehmenden (Vor-)Urteil entgegenwirken, das beste Controlling sei das ohne Controller. Sie können damit ihre Leistung mit der anderer Führungsdienstleister - z. B. der internen Revision oder auch der von fachlichen Stäben der Linien - vergleichen und in Folge ihre Position stärken. Schließlich ist - wie die Controller aus eigener Tätigkeit in anderen Unternehmensbereichen wissen - die Messung des Erfolgs stets ein großer Hebel, um anschließend den Erfolg zu steigern.

Den Erfolg des Controllings zu bestimmen, ist bei näherem Hinsehen allerdings nicht einfach. Geht es bei der Arbeit der Controller nicht darum, den Erfolg der Manager zu erhöhen? Ist die Frage nach einem eigenen Erfolg deshalb nicht schon im Kern falsch gestellt? Wie lässt sich der Erfolg von komplexen Dienstleistungen überhaupt messen? Gibt es dafür objektive Messgrößen (kann man etwa Benchmarkingwerten glauben?), muss man auf die Einschätzungen der Manager bauen? Wovon hängt der Erfolg der Controller ab? Wie kann er beeinflusst werden? Was unterscheidet erfolgreiche von nicht erfolgreichen Controllern?

Wir wollen in diesem Heft der ZfCM das Thema Controllingerfolg aus sehr unterschiedlichen Perspektiven beleuchten. Am Anfang steht ein Interview mit dem Pionier des Controllings in Deutschland, Albrecht Deyhle, der die Controlling-Entwicklung über einen langen Zeitraum maßgeblich geprägt hat und damit berufen ist, die Frage nach dem Controlling-Erfolg aus einer Gesamtperspektive heraus zu beantworten. Es schließt sich ein Beitrag aus meiner Feder an, der den Blick vom Controlling auf die Controller wendet und anhand unterschiedlicher empirischer Studien die Frage beantwortet, was erfolgreiche von weniger erfolgreichen Controllern unterscheidet. Andreas Hoffjan, Heinz-Werner Ufer und Anja Kolburg berichten anschließend über die Erkenntnisse von Experteninterviews, die sich um die Controllingeffizienz ranken und tiefe Einblicke in das geben, was Controller unter Controllingeffizienz verstehen und wie sie diese verbessern wollen. Im vierten und letzten Beitrag zum Schwerpunktthema stellen schließlich Stephanie Nachtsheim, Dirk Suwelack und Olaf Cunitz ein Projekt im Controlling der Bayer CropScience vor, das sowohl die operativen Controlling-Prozesse als auch das Controlling-Know-how nachhaltig verbessert hat.

Nicht nur in Krisenzeiten, in denen intensiv über den Abbau von Gemeinkosten nachgedacht wird, müsste dieses Heft somit von erheblicher praktischer Bedeutung sein - zumal es noch andere spannende Beiträge enthält, also quasi randvoll mit aktuellem Wissens ist. Viel Spaß und Nutzen beim Lesen!

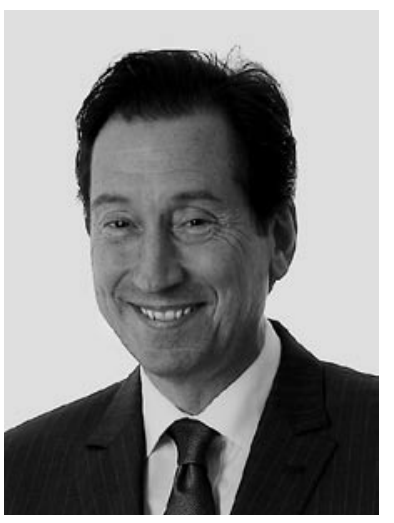

Jürgen Weber

$\mathrm{Ihr}$

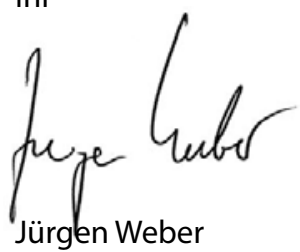

